\title{
DISTRIBUIÇÕES DE EXTREMOS DE PRECIPITAÇÃO DIÁRIA, TEMPERATURA MÁXIMA E MÍNIMA E VELOCIDADE DO VENTO EM PIRACICABA, SP (1917-2006)
}

\author{
CLÓVIS ANGELI SANSIGOLO \\ Centro de Previsão de Tempo e Estudos Climáticos (CPTEC), \\ Instituto Nacional de Pesquisas Espaciais (INPE) \\ clovis.sansigolo@cptec.inpe.br
}

Recebido Setembro 2007 - Aceito Março 2008

\begin{abstract}
RESUMO
Diversas distribuições teóricas de probabilidade incluindo a Normal, Gumbel, Fréchet, Weibull, LogNormal e Pearson 3, foram ajustadas aos extremos anuais de precipitação máxima diária, temperatura máxima e mínima absolutas e velocidade instantânea do vento em Piracicaba, SP (1917-2006). Métodos de máxima verossimilhança foram utilizados para estimar os parâmetros das distribuições, assim como seus intervalos de confiança. Testes $\chi 2$ e KS foram usados para comparar os ajuste e selecionar as melhores distribuições teóricas. Os ajustes também foram avaliados em gráficos PP e QQ. A distribuição de Gumbel foi a que melhor se ajustou aos extremos de precipitação máxima diária e de temperaturas máximas absolutas. As temperaturas mínimas absolutas apresentaram uma distribuição simétrica e foram melhor ajustadas pela distribuição normal. A distribuição de Weibull foi a que melhor se ajustou às velocidades instantâneas do vento. São apresentados resultados sobre os valores esperados, assim como seus intervalos de confiança, para períodos de retorno de até 500 anos.
\end{abstract}

Palavras-chave: Ajustes de distribuições de extremos; variáveis meteorológicas; Piracicaba, Brasil

\begin{abstract}
EXTREME DISTRIBUTIONS OF DAILY RAINFALL, MINIMUM AND MAXIMUM TEMPERATURES AND WIND SPEED IN PIRACICABA, BRAZIL (1917-2006).

Several theoretical probability distributions, including Normal, Gumbel, Fréchet, Weibull, Lognormal, and Pearson 3 were fitted to annual extremes of daily maximum rainfall, maximum and minimum absolute temperature, and instantaneous wind speed in Piracicaba, São Paulo, Brasil (1917-2006). Maximum likelihood methods were used to estimate the distributions parameters as well as their confidence intervals. KS and $\chi 2$ tests were used to compare the fits and select the best theoretical distribution. The Gumbel distribution was that best fitted the extremes of daily maximum rainfall and maximum absolute temperature. The minimum absolute temperatures presented a symmetrical distribution and were best fitted by the normal distribution. The Weibull distribution best fitted the maxima wind speeds. Results showing the expected values, as well as their confidence intervals, for return periods up to 500 years, are presented.
\end{abstract}

KeyWords: Extremes distributions fittings; meteorological variables; Piracicaba, Brazil

\section{INTRODUÇÃO}

Eventos raros ou extremos têm grande relevância na climatologia e hidrologia, e suas estimativas probabilísticas são imprescindíveis para o planejamento e desenvolvimento das atividades sujeitas a seus efeitos adversos, especialmente estruturas de engenharia civil e agricultura .

A teoria de valores extremos é fundamental nestes casos para a modelagem destes eventos. Os fundamentos desta teoria foram desenvolvidos por Fisher-Tippett (1928), que definiram os três tipos possíveis de distribuições assintóticas de valores extremos, conhecidas como de Gumbel (tipo I), Fréchet (tipo II) e Weibull (tipo III) (Gumbel, 1958), casos especiais da Distribuição Generalizada de Valores Extremos desenvolvida por Jenkinson (1955). Além das distribuições de valores extremos, também são bastante utilizadas para descrever eventos raros as distribuições Log-normal e Pearson 3 (Sevruk. \& Geiger, 1981).

Hershfield \& Kohler (1960) analisando os dados de milhares de estações pluviométricas nos Estados Unidos, concluíram que a distribuição de Gumbel é a mais adequada para estimar as probabilidades de ocorrência de eventos extremos de precipitação de diversas durações. 
No caso das velocidades máximas do vento, uma das distribuições mais utilizadas é a de Weibull ( Van der Auwera et al., 1980; Garcia et al., 1999 e Sansigolo, 2005).

Em relação aos extremos de temperatura máxima e mínima ainda não existe um consenso sobre distribuições preferenciais. Sansigolo \& Nery (2000) constataram que distribuições de Gumbel foram as que melhor se ajustaram às temperaturas mínimas de 27 localidades no Estado do Paraná.

Distribuições teóricas de probabilidade são simplesmente funções analíticas usadas para descrever o comportamento de determinadas variáveis. No caso de extremos, só o ajuste a séries longas em múltiplas localidades é que dá indicações sobre as distribuições que levam às melhores extrapolações.

Embora a teoria probabilística fundamental de valores extremos tenha sido desenvolvida há muito tempo, a modelagem estatística de extremos ainda permanece como assunto ativo de pesquisas dado seu importante papel nos projetos e gerenciamento de recursos hídricos, especialmente num contexto de mudanças climáticas (Katz et al., 2002).

O objetivo deste trabalho é uma análise crítica do ajuste de diferentes distribuições de extremos às longas séries de
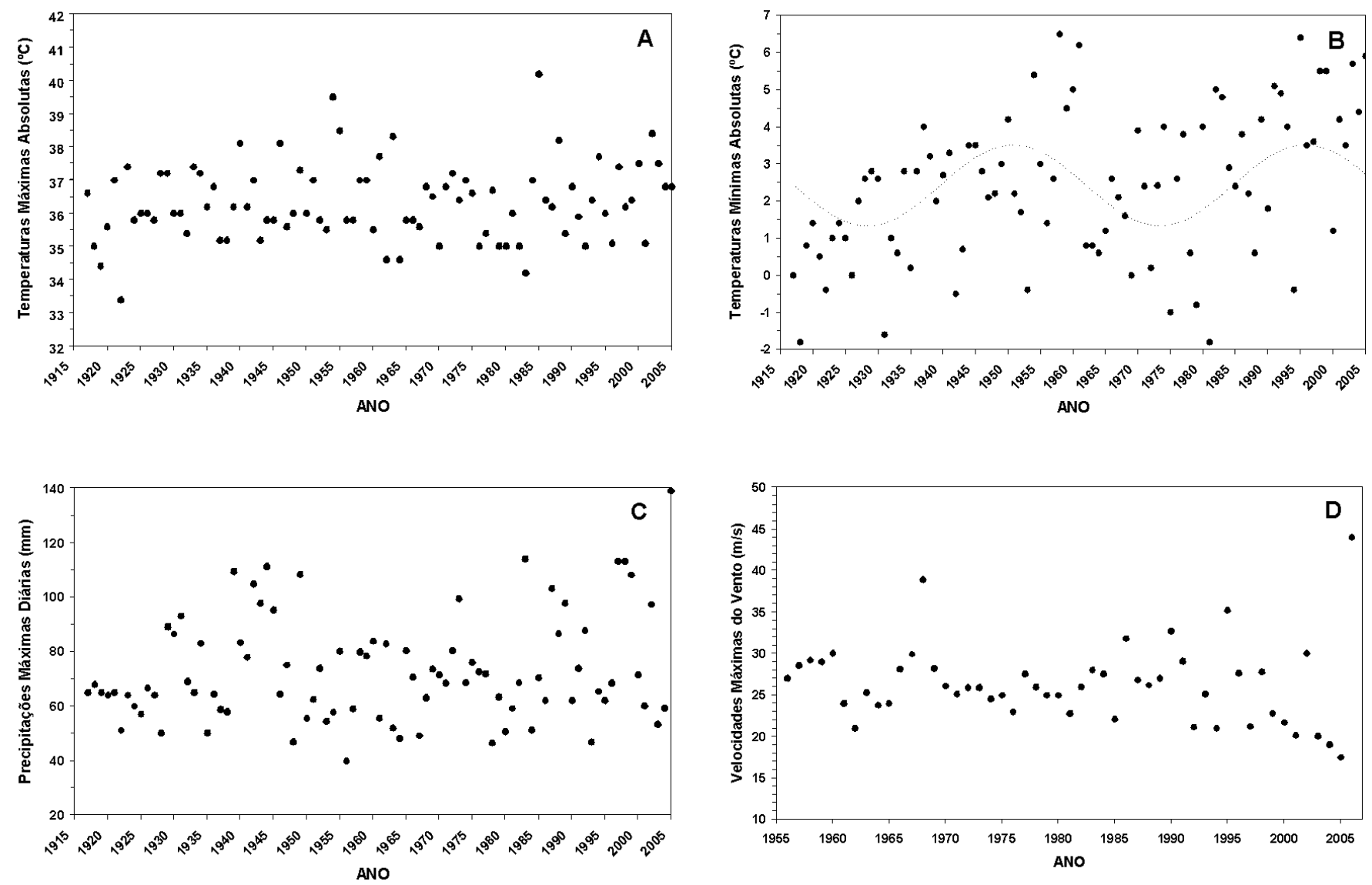

Figura 1. Séries temporais anuais dos extremos de temperatura máxima absoluta (A), mínima absoluta (B), precipitação máxima diária (C) e velocidade máxima instantânea do vento (D). 
Tabela 1. Temperaturas máximas e mínimas absolutas, precipitações máximas diárias e velocidades máximas do vento, assim como seus intervalos de confiança a 95\% de probabilidade, esperados com períodos de retorno entre 10 e 500 anos.

\begin{tabular}{|c|c|c|c|c|c|}
\hline \multirow[b]{3}{*}{ Temp. máx. abs. $\left({ }^{0} \mathrm{C}\right)$} & \multicolumn{5}{|c|}{$\begin{array}{c}\text { Valores esperados e seus respectivos intervalos de confiança } \\
\text { nos diversos tempos de retorno ( } \mathrm{T} \text {, anos) }\end{array}$} \\
\hline & $\mathrm{T}=10$ & $\mathrm{~T}=50$ & $\mathrm{~T}=100$ & $\mathrm{~T}=200$ & $\mathrm{~T}=500$ \\
\hline & $38,0 \pm 0,5$ & $39,5 \pm 0,9$ & $40,2 \pm 1,0$ & $40,9 \pm 1,1$ & $41,8 \pm 1,3$ \\
\hline Temp. mín. abs. $\left({ }^{0} \mathrm{C}\right)$ & $-0,1 \pm 0,6$ & $-0,8 \pm 0,6$ & $-2,2 \pm 0,8$ & $-2,7 \pm 0,9$ & $-3,3 \pm 0,9$ \\
\hline Prec. máx. diária (mm) & $95 \pm 6$ & $119 \pm 9$ & $129 \pm 10$ & $138 \pm 11$ & $151 \pm 13$ \\
\hline Vel. máx. do vento $\left(\mathrm{m} \cdot \mathrm{s}^{-1}\right)$ & $33,5 \pm 13$ & $35,2 \pm 14$ & $38,3 \pm 15,6$ & $39,4 \pm 16$ & $40,6 \pm 16,7$ \\
\hline
\end{tabular}
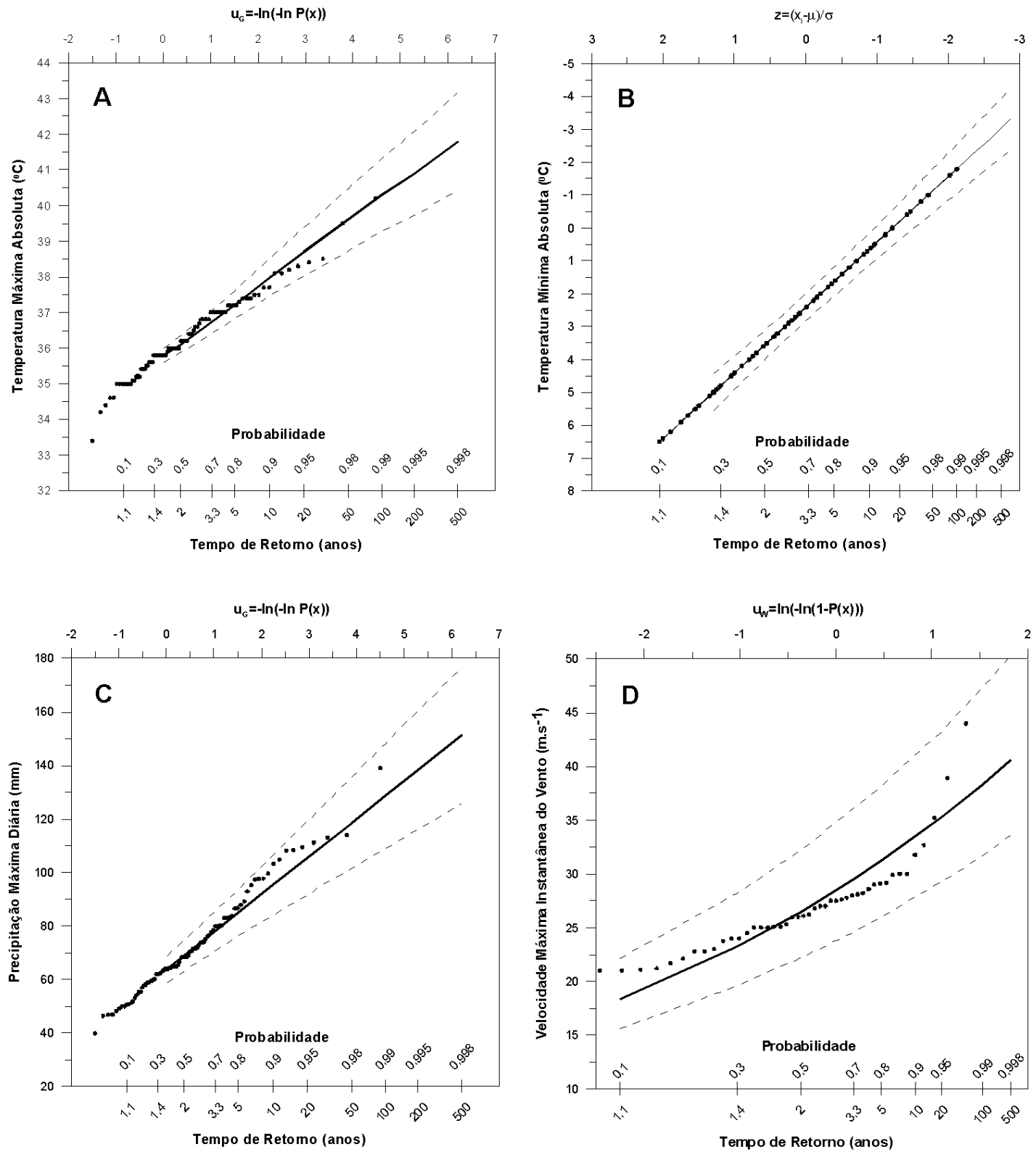

Figura 2. Distribuições de extremos ajustadas às temperaturas máximas absolutas (A), mínimas absolutas (B), precipitações máximas diárias (C) e velocidades máximas instantâneas do vento (D). Os pontos representam as observações, as linhas contínuas as curvas ajustadas e as tracejadas seus intervalos de confiança a $95 \%$ de probabilidade. 
consideradas, incluindo a de Gumbel, Log-Normal, Pearson 3, Fréchet e Weibull, que tem, respectivamente, como funções de densidade de probabilidade acumulada:

$$
\begin{array}{ll}
\mathrm{P}(\mathrm{x})_{\mathrm{G}}=\exp (-\exp (-\alpha(\mathrm{x}-\beta)) & \text { (Gumbel) } \\
\mathrm{P}(\mathrm{x})_{\mathrm{LN}}=1 / \beta \sqrt{2 \pi} \int_{-\infty}^{x} \exp ^{-0,5(\mathrm{x}-\mathrm{a}) / \beta]} \mathrm{dx}, & \text { (Log-Normal) } \\
\mathrm{P}(\mathrm{x})_{\mathrm{P} 3}=1 / \alpha \Gamma(\beta) \int_{0}^{x}((\mathrm{x}-\gamma) / \alpha)^{\beta-1} \exp ^{-(\mathrm{x}-\gamma) / \alpha} \mathrm{dx}, & \text { (Pearson 3) } \\
\mathrm{P}(\mathrm{x})_{\mathrm{FR}}=\exp (-\exp (-\alpha(\mathrm{x}-\beta)), & \text { (Fréchet) } \\
\mathrm{P}(\mathrm{x})_{\mathrm{W}}=1-\exp \left(-\alpha \mathrm{x}^{\beta}\right), & \text { (Weibull) }
\end{array}
$$

nas quais $\alpha, \beta$ e $\gamma$ são os parâmetros de posição, escala e forma (no caso das distribuições Log-normal e Frechet (Log-Gumbel) os parâmetros a, b são para a série transformada). Os parâmetros das distribuições foram ajustados por métodos de máxima verossimilhança (Kite, 1977), reconhecidos como os melhores estimadores. Também os intervalos de confiança foram obtidos por métodos de máxima verossimilhança (Kite, 1977).

Os ajustes e a seleção das melhores distribuições teóricas foram feitos por testes do Qui-Quadrado, que compara o efetivo observado e o teórico esperado em intervalos discretos e Kolmogov-Smirnov, que compara as distribuições empíricas acumuladas com as teóricas. Complementarmente, já que os testes do $\chi^{2}$ e KS são sómente adequados para a parte central das distribuições. Também foram utilizados gráficos PP (percentispercentis) e QQ (quantis-quantis) para avaliar o comportamento das distribuições nos seus limites.

\section{RESULTADOS E DISCUSSÃO}

Testes de Kendall aplicados às quatro séries temporais da figura 1 não detectaram tendências significativas ao nível de $95 \%$ de probabilidade em nenhuma delas. Portanto, mudanças climáticas globais ou regionais, que poderiam estar associadas por exemplo ao aquecimento urbano, não afetaram significativamente os registros. Ressalte-se que isto é em relação às intensidades dos eventos e não às suas freqüências que também podem ser afetadas por estas mudanças. O teste de Fisher estendido só detectou uma única periodicidade significativa a $95 \%$ de probabilidade na série das temperaturas mínimas absolutas. Este ciclo de 45 anos, que explica aproximadamente $14 \%$ da variância da série, é mostrado na figura $1 \mathrm{~b}$.

A distribuição de extremos que melhor se ajustou às temperaturas máximas absolutas foi a de Gumbel. Na figura 2a encontram-se as distribuições empírica e teórica acumulada $(\mathrm{P}(\mathrm{x})=\exp (-\exp (-1,109(\mathrm{x}-35,80)))$, assim como os intervalos de confiança ao nível $95 \%$ de probabilidade. Os valores obtidos para os testes de ajustamento do $\chi^{2}$ e KS foram, respectivamente, 0,09 e 5,6, inferiores aos valores críticos assintóticos para distribuições de extremos com $95 \%$ de probabilidade $(0,89$ e 9,5).

As temperaturas mínimas absolutas anuais apresentaram uma distribuição simétrica e não altamente assimétrica, como seria esperado no caso de extremos, logo foram melhor ajustadas pela distribuição normal $\left(\mathrm{P}(\mathrm{x})=1 / \sigma \sqrt{2 \pi} \int_{-\infty}^{x} \exp -0,5\right.$ $(\mathrm{x}-2,42) / 1,99])$, mostrada na figura $2 \mathrm{~b}$. Os valores obtidos para os teste do $\chi^{2}$ e KS foram, respectivamente, 0,09 e 2,8, inferiores aos valores críticos a $95 \%(0,89$ e 9,5$)$. Isto pode ser explicado pela presença do ciclo de 45 anos na série.

As precipitações diárias máximas anuais também foram melhor ajustadas pela distribuição de Gumbel. Na figura 2c encontram-se as distribuições empírica e a teórica acumulada $(\mathrm{P}(\mathrm{x})=\exp (-\exp (-0,069(\mathrm{x}-63,84)))$, assim como os intervalos de confiança ao nível $95 \%$ de probabilidade. Os valores encontrados para os testes do $\chi^{2}$ e KS foram, respectivamente, 0,07 e 3,3, muito inferiores aos valores críticos a $95 \%$ de probabilidade $(0,89$ e 12,6). Este resultado confirma que a distribuição de Gumbel é a mais adequada para descrever e extrapolar extremos de precipitação diária para longos períodos de retorno (Hersfield $\&$ Kohler, 1960).

As velocidades instantâneas máximas anuais do vento a $10 \mathrm{~m}$ de altura foram melhor ajustadas pela distribuição de Weibull ( $\mathrm{P}(\mathrm{x})=1-\exp \left(-3,085 \cdot 10^{-8} \mathrm{x}^{5,163}\right)$, mostrada na figura $2 \mathrm{~d}$. Os valores obtidos para os teste do $\chi^{2}$ e KS foram, respectivamente, 0,1 e 3,7, inferiores aos valores críticos a 95\% $(0,89$ e 7,8$)$. Este resultado confirma que esta distribuição é a mais apropriada não só para descrever os extremos de vento, como para extrapolá-los para longos períodos de retorno (Van der Auwera et al. 1980; Garcia et al. 1998, Bautista, 2002 e Sansigolo, 2005).

Os valores esperados de temperaturas máximas e mínimas absolutas, precipitações máximas diárias e velocidades máximas do vento para períodos e retorno de 10 a 500 anos, assim como seus intervalos de confiança a $95 \%$ de probabilidade, encontram-se na tabela 1 .

Ressalta-se na tabela 1, que os grandes intervalos de confiança associados às estimativas das velocidades máximas do vento, podem parcialmente ser explicados pelo menor tamanho desta série (50 anos) em relação às outras três (90 anos).

Temperaturas mínimas absolutas de $30 \mathrm{C}$ no abrigo $\left(\approx 0^{0} \mathrm{C}\right.$ na superfície) ou o risco de pelo menos uma geada por ano, é da ordem de $61 \%$, correspondente a um tempo de retorno de 2,6 anos

Como os testes de ajustamento do Qui-quadrado e Kolmogorov-Smirnov são insensíveis nos extremos das distribuições e testes alternativos satisfatórios não existem, gráficos $\mathrm{PP}$ e QQ, mostrados na figura 3, foram complementarmente usados para avaliar o comportamento das distribuições nos seus extremos. Verifica-se que o gráfico QQ 

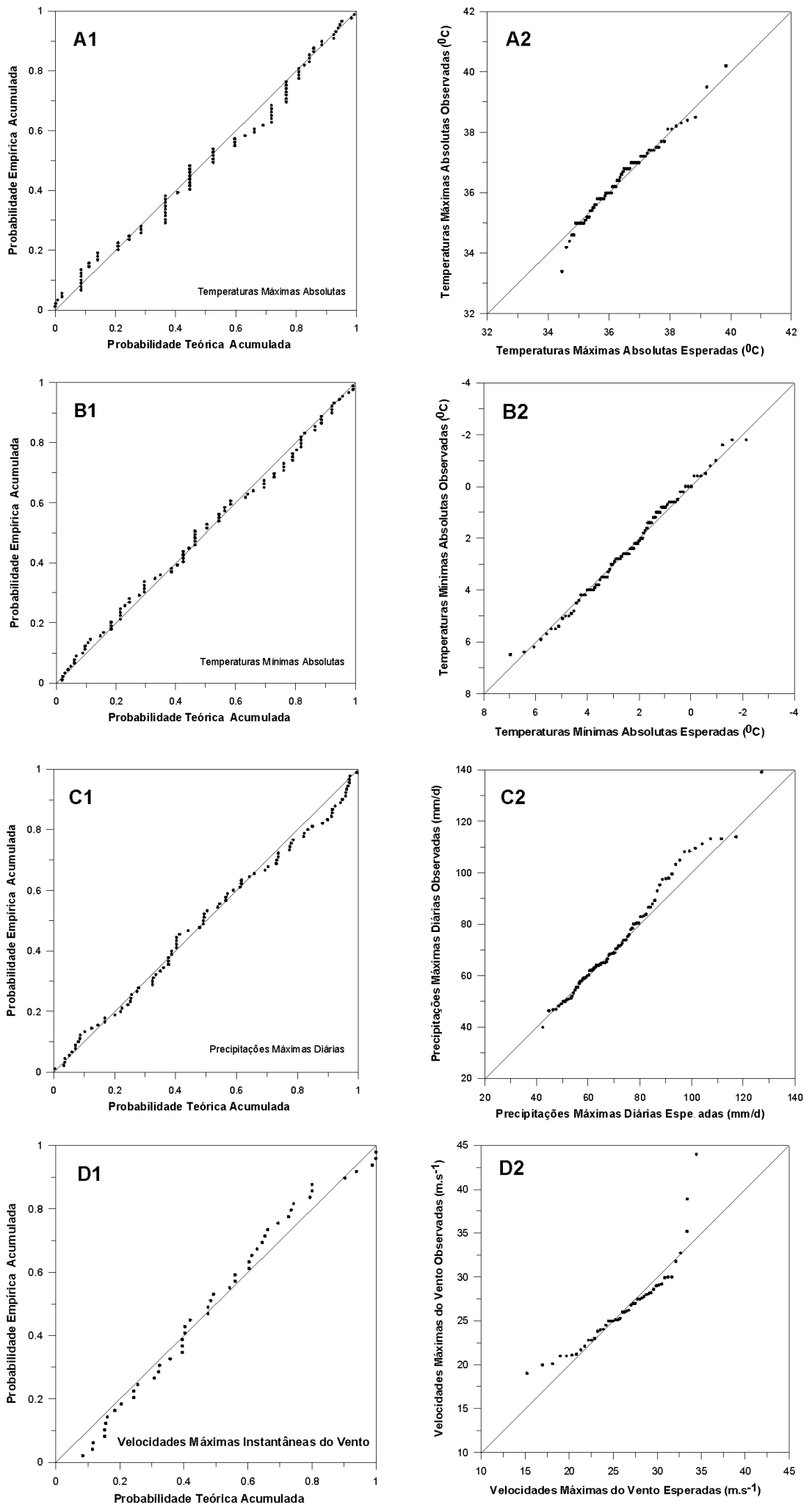

Figura 3 - Gráficos PP e QQ para as temperaturas máximas absolutas (A1 e A2), Mínimas absolutas (B1 e B2), Precipitações máximas diárias (C1 e C2) e Velocidades máximas do vento (D1 e D2) 
das velocidades máximas do vento (Fig. 3 D2) mostra uma falta de ajuste do modelo para valores superiores a $35 \mathrm{~m} . \mathrm{s}^{-1}$, levando a sub-estimativas nesta região.

Séries temporais longas possibilitam melhores ajustes e verificações, mas por outro lado podem estar contaminadas pela presença de tendências e ciclos de longo prazo, que devem ser previamente analisados.

\section{CONCLUSÕES}

Tendências significativas, que poderiam estar associadas com mudanças climáticas, não foram constatadas em nenhuma das quatro séries de extremos analisadas. A série das mínimas absolutas apresentou uma única periodicidade significativa de 45 anos.

Estimativas de risco de eventos extremos são processos complexos que envolvem ajustes e verificação de diferentes distribuições teóricas de probabilidade. A decisão sobre a melhor distribuição deve envolver estimadores, intervalos de confiança, testes de ajustamento e gráficos P-P e Q-Q para avaliar o comportamento das distribuições nos seus limites.

As distribuições teóricas de probabilidade que melhor se ajustaram aos extremos de temperatura máxima e mínima absoluta, precipitação máxima diária e velocidade máxima do vento foram, respectivamente, Gumbel, Normal, Gumbel e Weibull.

Como os ajustes estão baseados em séries longas e homogêneas, exceto no caso das Temperaturas mínimas anuais, estas distribuições podem ser usadas com bastante confiabilidade para extrapolação em períodos mais longos, desde que não ocorram futuras variações climáticas significativas na região.

\section{REFERÊNCIAS BIBLIOGRÁFICAS}

BAUTISTA, E. Z. A distribuição generalizada de valores extremos no estudo da velocidade máxima do vento em Piracicaba, SP. Dissertação de Mestrado, ESALQ, USP, Piracicaba, 47p. 2002.
GARCIA, A.; TORRES, J. L.; PRIETO, E.; FRANCISCO, A. Fitting Wind Speed Distributions: A Case Study. Solar Energy, v. 6, n. 2, p. 139-144, 1998.

GUMBEL, E. J. Statistics of Extremes. Columbia University Press, New York, 375p. 1958.

HERSFIELD, D. M.; KOHLER, M. A,An empirical appraisal of the Gumbel extreme value procedure. J. Geophys. Res., v. 65 , n. 6 , p. $1737-1746,1960$.

JENKINSON, A. F. The frequency distribution of the annual maximum (or minimum) values of meteorological elements. Q. J. R. Met. Soc., v. 81, p. 159-171, 1955.

KATZ, R. W.; PARLANGE, M. B.; NAVEAU, P. Statistics of extremes in hydrology. Advances in Water Resources, n. 25, p. 1287-1304., 2002.

KITE, G. W. Frequency and Risk Analysis in Hydrology. Water Resources Publications, Fort Collins, CO, 224 p. 1977.

SANSIGOLO, C. A.; NERY, J. T. Distribuições de extremos de temperatura mínima no Paraná. Rev. Bras. Agromet., v. 8, n. 2, p. 247-253, 2000.

SANSIGOLO, C. A. Distribuições de probabilidade de velocidade e potência do vento. Rev. Bras. Met., v. 20, n. 2, p. 207-214, 2005.

SEVRUK, B.; GEIGER, H. Selection of distribution types for extremes of precipitation. WMO Operational Report, $n$. 15, 64p. 1981.

SHIMSHONI, M. On Fisher's test of significance in harmonic analysis. Geophys. J. Roy. Astronom. Soc., v. 23, p.373377, 1971.

SNEYERS, R. Sur l'analyse statistique des séries d'observations. OMM Note Technique, n. 143, 192p. 1975.

VAN DER AUWERA, L.; MEYER, F.; MALET, L.M. The Use of the Weibull 3-Parameters model for estimating mean wind power densities. J. Appl. Meteorol., v. 19, n. 7, p. $819-825,1980$. 